\title{
Limb Remote Ischemic Conditioning Ameliorates Cognitive Impairment in Rats with Chronic Cerebral Hypoperfusion by Regulating Glucose Transport
}

\author{
Changhong Ren 1,5,\#, Yuanyuan Liu ${ }^{1,2, \#, ~ C h r i s t o p h e r ~ S t o n e ~}{ }^{4}$, Ning Li ${ }^{1,5}$, Sijie Li ${ }^{1}$, Haiyan Li ${ }^{1,5}$, \\ Zichao Cheng', ${ }^{1,3}$, Jiangnan $\mathrm{Hu}^{6}$, Weiguang $\mathrm{Li}^{1}$, Kunlin Jin ${ }^{7}$, Xunming Ji ${ }^{*}$, Yuchuan Ding1,4
}

${ }^{1}$ Beijing Key Laboratory of Hypoxia Translational Medicine, Xuanwu Hospital, Capital Medical University, Beijing, China. ${ }^{2}$ Department of Endocrinology, The Affiliated Huai'an First People's Hospital of Nanjing Medical University, Huai'an, China. ${ }^{3}$ Department of Rehabilitation Medicine, Affiliated 3201 Hospital of Xi'an Jiaotong University School of Medicine, Hanzhong, China. ${ }^{4}$ Department of Neurosurgery, Wayne State University School of Medicine, Detroit, MI 48201, USA. ${ }^{5}$ Beijing Institute of Brain Disorders, Capital Medical University, Beijing, China. ${ }^{6}$ Department of Pharmaceutical Sciences, University of North Texas Health Science Center, Fort Worth, Texas 76107, USA. ${ }^{7}$ Department of Pharmacology \& Neuroscience, University of North Texas Health Science Center, Fort Worth, Texas 76107, USA.

[Received September 24, 2020; Revised November 12, 2020; Accepted November 14, 2020]

\begin{abstract}
Cognitive impairment is closely associated with the slowing of glucose metabolism in the brain. Glucose transport, a rate-limiting step of glucose metabolism, plays a key role in this phenomenon. Previous studies have reported that limb remote ischemic conditioning (LRIC) improves cognitive performance in rats with chronic cerebral hypoperfusion $(\mathrm{CCH})$. Here, we determined whether LRIC could ameliorate cognitive impairment in rats with $\mathrm{CCH}$ by regulating glucose transport. A total of 170 male Sprague-Dawley rats were used. Animals subjected to permanent double carotid artery occlusion (2VO) were assigned to the control or LRIC treatment group. LRIC was applied beginning 3 days after the 2 VO surgery. We found that LRIC can improve learning and memory; decrease the ratio of ADP/ATP; increase glucose content; upregulate the expression of pAMPK $\alpha$, GLUT1 and GLUT3; and increase the number of GLUT1 and GLUT3 transporters in cerebral cortical neurons. The expression of GLUT1 and GLUT3 in the cortex displayed a strong correlation with learning and memory. Pearson correlation analysis showed that the levels of GLUT1 and GLUT3 are correlated with neurological function scores. All of these beneficial effects of LRIC were ablated by application of the AMPK inhibitor, dorsomorphin. In summary, LRIC ameliorated cognitive impairment in rats with $\mathrm{CCH}$ by regulating glucose transport via the AMPK/GLUT signaling pathway. We conclude that AMPK-mediated glucose transport plays a key role in LRIC. These data also suggest that supplemental activation of glucose transport after CCH may provide a clinically applicable intervention for improving cognitive impairment.
\end{abstract}

Key words: ovary, aging, pathophysiology, limb remote ischemic conditioning, cognitive impairment, glucose transport, adenosine monophosphate activated protein kinase, chronic cerebral hypoperfusion

Vascular dementia $(\mathrm{VaD})$ is type of major neurocognitive disorder caused by hypoxic or hemorrhagic brain tissue damage that accumulates over time due to various acute and chronic cerebrovascular diseases [1,2]. Numerous studies have demonstrated that chronic cerebral hypoperfusion $(\mathrm{CCH})$ is associated with the initiation and progression of both $\mathrm{VaD}$ and Alzheimer's disease (AD). $\mathrm{VaD}$ is the second most common type of dementia after

*Correspondence should be addressed to: Dr. Xunming Ji, Beijing Institute of Brain Disorders, Capital Medical University, Beijing 100053, China. E-mail: jixm@ccmu.edu.cn. \#These authors equally contributed this work.

Copyright: () 2020 Ren C et al. This is an open-access article distributed under the terms of the Creative Commons Attribution License, which permits unrestricted use, distribution, and reproduction in any medium, provided the original author and source are credited. 
$\mathrm{AD}$, accounting for $20 \%$ of all dementias; moreover, as society continues to age, this proportion is projected to triple by 2050 [3]. Unfortunately, despite this serious and increasing contribution to neurocognitive morbidity, the capacity to treat $\mathrm{VaD}$ is very limited. Beyond standard measures to control vascular risk factors such as hypertension and diabetes, only a small number of cholinesterase inhibitors have been clinically proven to be effective and even these only have modest improvement in cognitive function while failing to modify overall prognosis [4].

In this setting, there is an urgent need to discover and perfect new therapies designed to prevent the onset of $\mathrm{VaD}$, ameliorate its progression, or both. One method that possesses potential to accomplish these goals is limb remote ischemic conditioning (LRIC). LRIC entails instituting a transient sublethal blood flow blockage of the distal limb that has been shown to induce endogenous protection and protect vital organs (such as the heart, brain, kidneys, etc.) from severe fatal ischemic injury [5]. As it gains recognition as an unconventional means to prevent and treat hypoxic-ischemic cerebrovascular diseases, LRIC has attracted the attention of an increasing number of research groups from all over the world. LRIC can effectively improve collateral circulation, increase cerebral blood flow in ischemic brain regions and reduce the recurrence rate of stroke [6-8]. In a recent study of relevance to $\mathrm{VaD}$ specifically, Wang et al. found that after one year of LRIC treatment, patients with cerebral small vessel disease and mild cognitive impairment had increased learning and memorization abilities along with significantly reduced volume of white matter degeneration as measured by brain MRI [9]. In a rodent model of focal cerebral ischemia, LRIC reduced infarct volume, promoted functional recovery after cerebral ischemia and improved cognitive functions [10-12]. Although it has been further reported that LRIC can improving learning and spatial memory in rodents and patients $[7,9,13,14]$, the exact mechanism of this improvement has not been fully elucidated.

Vascular diseases function in a multifaceted manner to cause chronic ischemia and hypoxia of the brain tissue, which in turn results in metabolic disorders [15], oxidative stress [16] and blood-brain barrier destruction [17, 18] among other deleterious changes including the induction of damage in neurons and white matter [14], all of which ultimately converge on the development of $\mathrm{VaD}$. In the nervous system, the aerobic metabolism of glucose is the main source of energy in the form of adenosine triphosphate (ATP) $[15,19,20]$; consequently, any defects in or obstacles to glucose metabolism will precipitate a decline in cognitive function [21, 22]. The glucose transporter (GLUT) is especially important in this context, as it has been proven to be the rate-limiting step of glucose metabolism [19, 23]. In the mammalian brain, GLUT1 and GLUT3 are the predominant GLUTs responsible for glucose transport [24]. Mice with reduced GLUT1 levels display an age-dependent decrease in cerebral blood flow, glucose uptake and cognitive function [21]. Similarly, decreased GLUT1 and GLUT3 have been observed in the $\mathrm{AD}$ brain in humans and contribute to the impairment of brain glucose uptake/metabolism [25, 26]. Adenosine monophosphate activated protein kinase (AMPK) is involved in this process as a molecular center for the control of cell energy metabolism. It is found widely distributed in brain tissues and is responsible for controlling the level of energy metabolism in cells by regulating the expression of GLUT genes [27] and its accumulation was found to be associated with a protective effect conferred by remote ischemic conditioning against cerebral hemorrhage [28].

To date, despite the considerable plausibility generated by previous work, no direct evidence that LRIC promotes learning and memory associated with AMPKmediated glucose uptake in $\mathrm{CCH}$, has been shown. Consequently, the implications of this promising new therapy for the treatment of $\mathrm{VaD}$ have not been fully explored. In this study, we attempted to explore these implications by using a $\mathrm{CCH}$ rat model subjected to LRIC and evaluate the impact of LRIC on cognitive impairment through the regulation of glucose transport via the AMPK/GLUT signaling pathway.

\section{MATERIALS AND METHODS}

\section{Animals}

All animal experiments were approved by the Animal Care and Use Committee of Xuanwu Hospital, Capital Medical University, China and conducted according to National Institutes of Health guidelines. Adult male Sprague-Dawley rats (220 to $260 \mathrm{~g}$ in weight) were purchased from Vital River Laboratories, Beijing, China and maintained on a 12-hour light/dark cycle with unlimited access to food and water.

\section{CCH model}

The $\mathrm{CCH}$ model was established using the double carotid artery occlusion (2VO) model, as described previously [29]. In brief, rats were anesthetized with $4 \%$ enflurane and maintained in an anesthetic state with a mixture of $70 \% \mathrm{~N}_{2} \mathrm{O}$ and $30 \% \mathrm{O}_{2}$ containing $1.5-2 \%$ enflurane using a small animal anesthesia system. The anesthetized rats were fixed on the operating table in the supine position. After routine skin preparation, an incision was cut on the skin in the middle of the neck. The bilateral common carotid arteries were carefully separated from the cervical 
sympathetic and vagus nerves. One carotid artery was then ligated with a silk thread. After 15 minutes, the contralateral carotid artery was also ligated. Finally, the neck incision was sutured. During the surgery, rectal temperature was monitored and maintained at $37 \pm 0.5^{\circ} \mathrm{C}$ with a heat blanket. Rats in the sham group underwent the same surgical procedure except that the carotid arteries were not ligated.

\section{LRIC treatment}

LRIC was performed as previously described [8] with some modifications. LRIC treatment started on the third day after $2 \mathrm{VO}$ surgery and was administered once daily until tissue collection. First, rats were anesthetized with sodium pentobarbital $(30 \mathrm{mg} / \mathrm{kg})$ intraperitoneally. Selfdeveloped rat LRIC instruments were then used (Patent No. ZL201720524103.7); rat limb cuffs were wrapped on each hind limb and LRIC was performed for three cycles at $10 \mathrm{mins} / \mathrm{cycle}$ at $240 \mathrm{mmHg}$ with intervening 10-min reperfusion intervals. Rats in the sham and $2 \mathrm{VO}$ groups were anesthetized similarly but did not undergo LRIC treatment thereafter. LRIC is implemented once a day until the animal is euthanized.
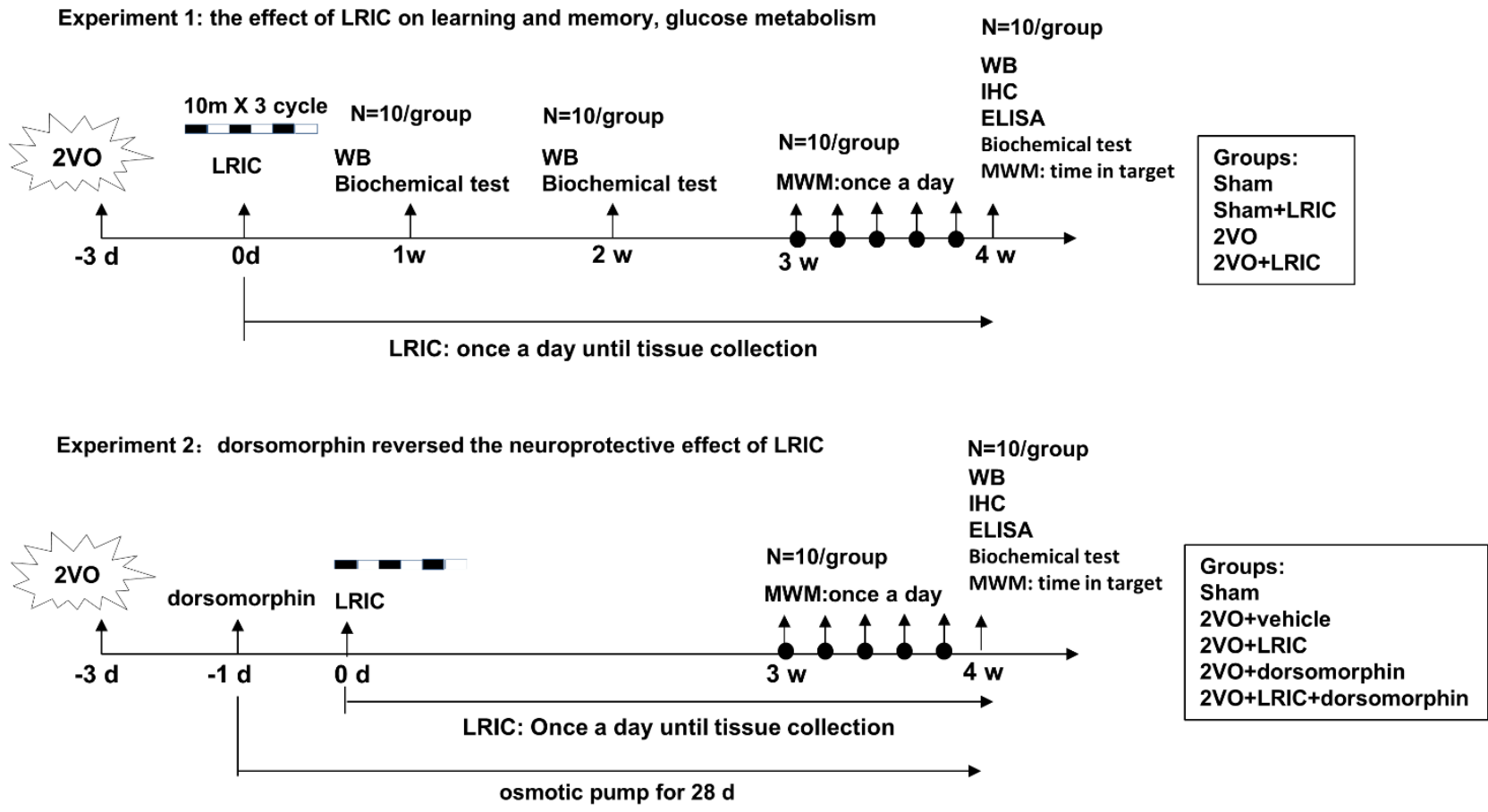

Figure 1. Experimental design and animal groups. 2VO, double carotid artery occlusion; WB, Western blot; ELISA, Enzyme-linked immunosorbent assay; IHC, Immunohistochemistry; MWM, Morris water maze; LRIC, Limb remote ischemic conditioning.

\section{Experimental design}

Animals were divided into groups for two experimental studies in a randomized fashion using Excel-generated random numbers and experiments were performed in a blinded manner (Fig. 1). Experiment 1: To evaluate the effect of LRIC on learning and memory, and glucose metabolism, rats were randomly divided into the following groups: (i) sham, (ii) sham+LRIC, (iii) $2 \mathrm{VO}$ and (iv) 2VO+LRIC. Experiment 2: To explore the underlying mechanisms of LRIC on glucose transport, we administered dorsomorphin, a selective AMPK inhibitor. Rats were randomly assigned to the following groups: (i) sham, (ii) 2VO+vehicle, (iii) 2VO+LRIC, (iv) 2VO+dorsomorphin and (v) 2VO+LRIC+dorsomorphin.

\section{Morris water maze}

The spatial learning and memory abilities of the rats were evaluated using the Morris water maze (MWM, DMS-2, Beijing, China) on the 21st day (i.e., after 3 weeks of LRIC treatment), as described previously [7]. The water maze used in our study was a flat, black, galvanized metal tank with a diameter of $150 \mathrm{~cm}$ and a height of $50 \mathrm{~cm}$, within which a platform of $10 \mathrm{~cm}$ in diameter and $23 \mathrm{~cm}$ in height was located. The tank contained $25 \mathrm{~cm}$ of water and the platform was placed $1-2 \mathrm{~cm}$ below the surface of 
the water. The training maze was not visible. The maze was divided into four equal quadrants (I, II, III, IV). In addition, a fixed visual cue (i.e., paper attached to the wall) was provided in the room. A camera was located above the center of the maze to record the movement of the rats and to send the data generated from this movement to an automatic tracking system (Noldus, EthoVision-XT 11).

The water maze experiment was completed within 6 days. For the first 5 days, the platform was fixed in the center of quadrant IV and rats were placed at different locations in the tank to train four times per day for 120seconds sessions. If the rats did not find the platform within $120 \mathrm{~s}$, they were picked up and placed on the platform for 20 seconds; if they did find it within $120 \mathrm{~s}$, they were immediately removed from the platform. On the sixth day, an exploratory test was performed; the platform was removed, and the rats were allowed to swim and search for the platform for $60 \mathrm{~s}$. All data were recorded using the EthoVision-XT 11 software, including the time spent to look for the target quadrant, the frequency with which rats crossed the position formerly occupied by the platform, the speed of swimming and the distance covered. The observer was blinded to the experimental conditions while performing the Morris water maze.

\section{Tissue collecting and processing}

After 1, 2 and 4 weeks of LRIC treatment, rats were anesthetized by intraperitoneal administration with 400 $\mathrm{mg} / \mathrm{kg}$ chloral hydrate and then perfused with saline. Left hemispheric tissues were taken, fixed in 4\% PFA for $24 \mathrm{~h}$ and stored in sucrose solution (30\% PBS) at $4{ }^{\circ} \mathrm{C}$ for 48 $\mathrm{h}$. Then, brain tissues were embedded in a medium for frozen tissue specimens and stored at $-80{ }^{\circ} \mathrm{C}$ for immunohistochemical staining. The right hemispheric cortex was immediately removed and placed on ice. Cortical tissues were then carefully separated and stored at $-80{ }^{\circ} \mathrm{C}$ for biochemical, ELISA, and Western blot analyses.

\section{Biochemical testing}

The ratio of ADP/ATP in the rat cortex homogenate was determined using a chemiluminescence kit (SigmaAldrich, Saint Louis, USA) according to the manufacturer's protocol. The contents of glucose, lactic acid and pyruvic acid were determined using detection kits (Nanjing Jiancheng Biotechnology Co., Ltd, Nanjing, China) according to the manufacturer's protocol as well. All assays were performed in triplicates. The observer was blinded to the experimental conditions.

C

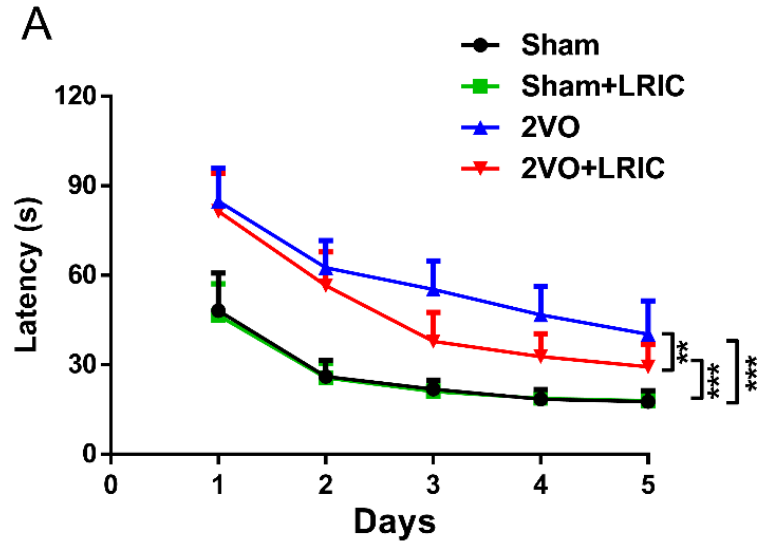

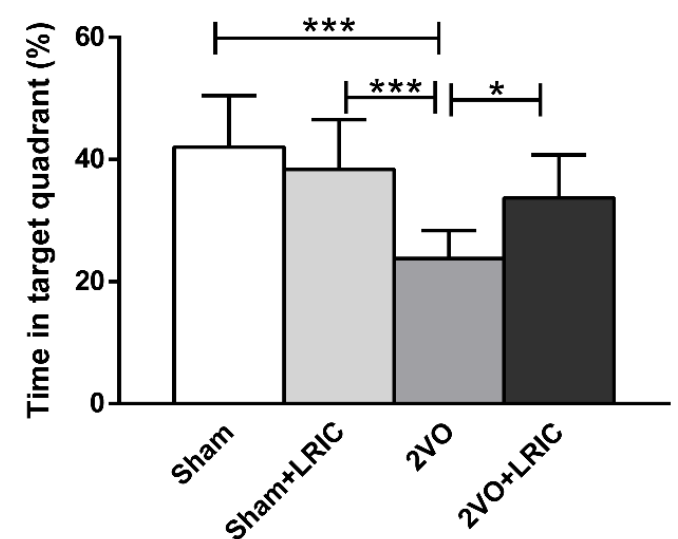

Figure 2. LRIC improved learning and memory in 2VO rats using the Morris water maze. (A) Escape latency time determined during Morris water maze testing beginning 3 weeks after LRIC treatment. (B) Percentage of time spent in the target quadrant. $* P<0.05, * * P<0.01, * * * P<0.001, \mathrm{n}=10 /$ group.

\section{Western blot}

The stored cerebral cortex tissues were weighed and then lysed in RIPA buffer using an ultrasonicator. The supernatant was extracted, and protein concentration was determined using the BCA method. A total of $60 \mu \mathrm{g}$ of protein was separated using SDS-PAGE and transferred onto a PVDF membrane. The membrane was blocked at room temperature for $1 \mathrm{~h}$ and incubated overnight at $4{ }^{\circ} \mathrm{C}$ with primary antibodies against GLUT1, GLUT3 and pAMPK $\alpha$ (Cell Signaling, USA). The specific reaction was visualized using a chemiluminescent substrate (GE Healthcare, UK) and $\beta$-actin was used to verify equal loading (1:3000, Sigma-Aldrich, Missouri, USA). The optical density of protein was measured using ImageJ software (NIH, Bethesda, MD, USA). 
A

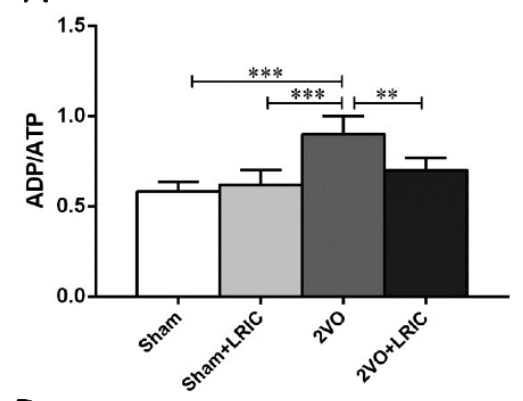

D

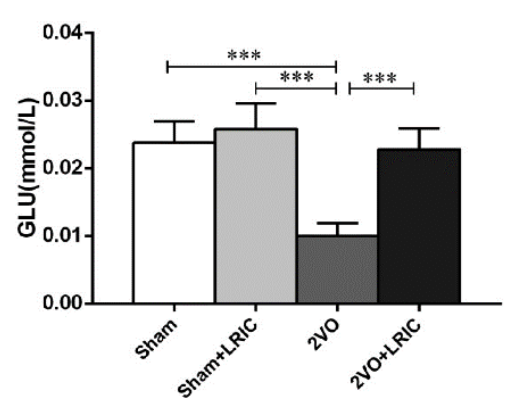

B

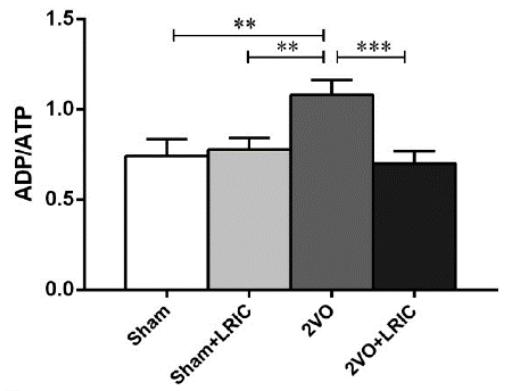

$\mathrm{E}$

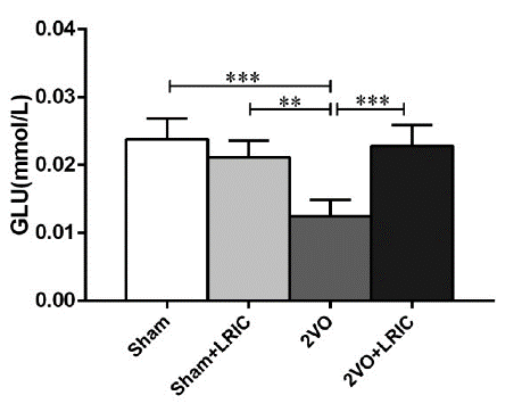

C

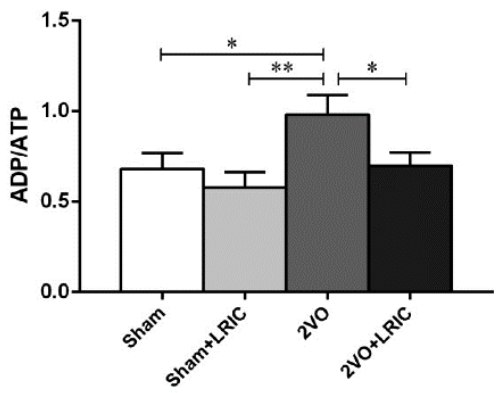

$\mathrm{F}$

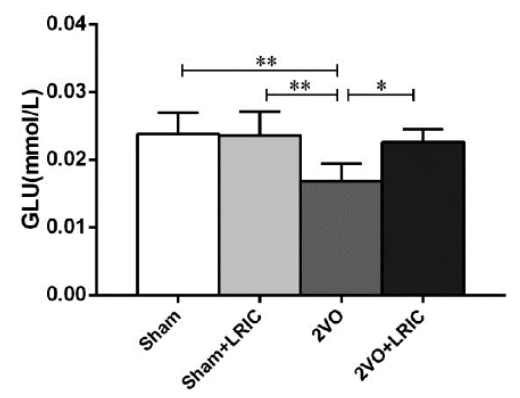

Figure 3. LRIC increased the content of ATP and glucose in the cortex of $2 \mathrm{VO}$ rats. (A and D) show the ratio of ADP/ATP and glucose content after 1 week of treatment. (B and E) show the ratio of ADP/ATP and glucose content after 2 weeks of treatment. ( $\mathbf{C}$ and $\mathbf{F}$ ) show the ratio of ADP/ATP and glucose content after 4 weeks of treatment. $* \mathrm{P}<0.05, * * \mathrm{P}<0.01, * * * \mathrm{P}<0.001$. Bar graphs are mean $\pm \mathrm{SD}, \mathrm{n}=10$ /group.

\section{Enzyme linked immunosorbent assay (ELISA)}

The contents of GLUT1 and GLUT3 in the cortex were determined using ELISA kits (EK14283, EK14285, respectively, Signalway antibody). The cerebral cortex was homogenized in $5 \mathrm{~mL}$ of PBS with a glass homogenizer on ice. The resulting suspension was sonicated with an ultrasonic cell disrupter. Thereafter, the homogenates were centrifugated for $5 \mathrm{mins}$ at $5000 \times \mathrm{g}$. The supernatant was assayed according to the manufacturer's protocol. The observer was blinded to the experimental conditions.

\section{Immunofluorescence staining}

Frozen brain tissues were sliced into $10 \mu \mathrm{m}$ sections and prepared for immunostaining as described previously [30]. The primary antibodies were rabbit anti-NeuN (1:300, Invitrogen, USA), mouse anti-GLUT1 and mouse anti-GLUT3 antibodies (1:400, Santa Cruz, USA). The secondary antibodies were Alexa Fluor 488-, 594conjugated donkey anti-mouse or anti-rabbit, (1:200; Invitrogen, Grand Island, NY, USA). Slides were mounted using ProLong Gold antifade reagent with DAPI (Molecular Probes, USA) and observed with a Nikon Ti
Eclipse Epi-Fl Illuminator (Nikon, Japan). The images were taken from six fields in the cerebral cortex. The observer was blinded to the experimental conditions.

\section{Intracerebroventricular injection with osmotic pump}

Intracerebroventricular administration and embedding of the osmotic pump were performed as previously described [31, 32]. Briefly, rats were placed in a stereotaxic apparatus under $2.5 \%$ enflurane anesthesia. A 28-day Alzet osmotic pump (Alza Corporation, Palo Alto, CA, USA) was implanted in a subcutaneous pocket on the back of the rats. A catheter connected to the pump was inserted into the lateral ventricle at the following coordinates based on the location of the bregma: anteroposterior $1 \mathrm{~mm}$, right lateral $1.5 \mathrm{~mm}$ and depth 3.5 $\mathrm{mm}$. The AMPK-specific inhibitor, dorsomorphin (0.1 $\mu \mathrm{mol} /$ day, purity $\geq 98 \%$, P5499, Sigma-Aldrich, St. Louis, MO, USA), was dissolved in $20 \%$ DMSO in PBS. The Alzet pumps then delivered either dorsomorphin or the vehicle at a rate of $1 \quad \mathrm{l} / \mathrm{h}$ over 28 days. Treatment was blinded to the operator and randomized before surgery by drawing lots. Drug administration was maintained during behavioral tests. 

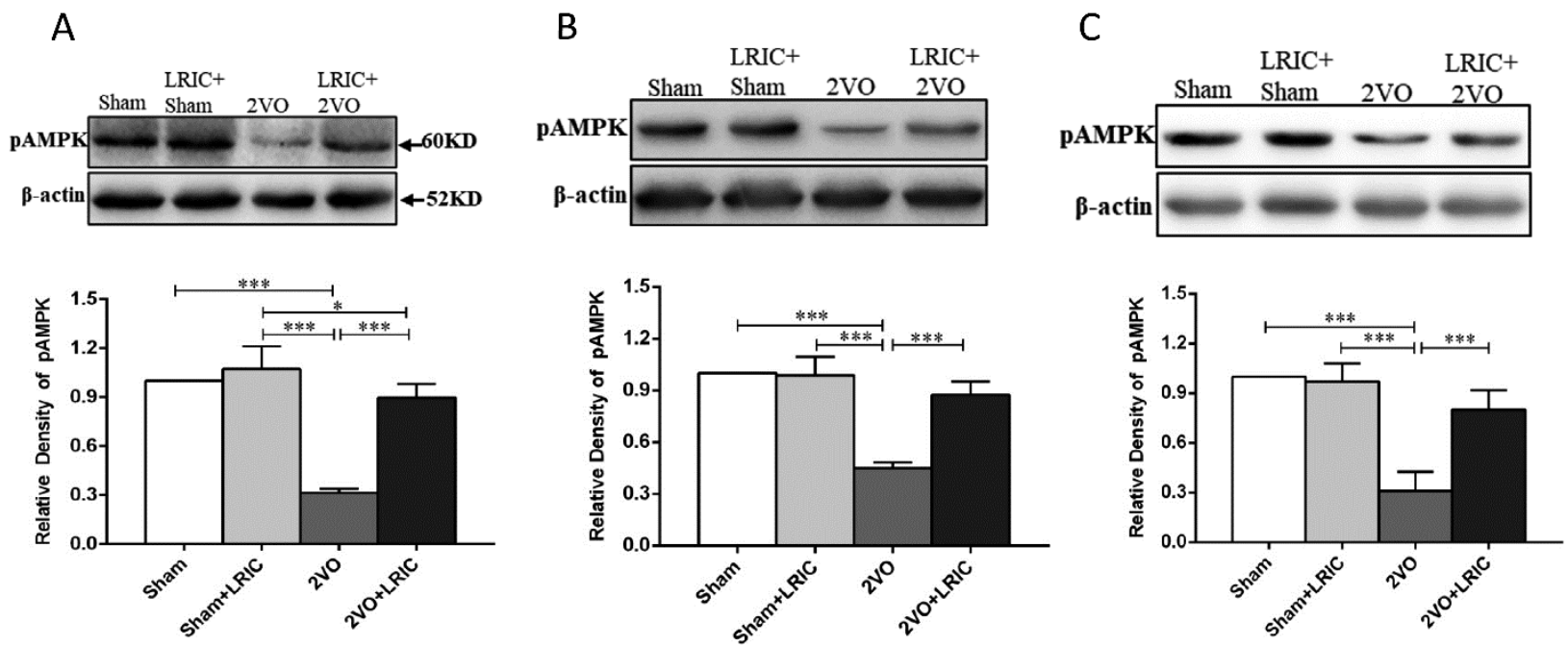

Figure 4. LRIC increased levels of PAMPK in the cerebral cortex as detected by Western blotting. (A and D) show levels of pAMPK $\alpha$ at 1 week after treatment initiation. (B and $\mathbf{E}$ ) show levels of pAMPK $\alpha$ at 2 weeks after treatment initiation. (C and $\mathbf{F})$ show levels of pAMPK $\alpha$ at 4 weeks after treatment initiation. $* P<0.05, * * P<0.01, * * * P<0.001$. Bar graphs are mean $\pm \mathrm{SD}, \mathrm{n}=10 / \mathrm{group}$.

\section{Statistical analysis}

All data are expressed as mean \pm standard deviation. Escape latencies were compared using repeated measures ANOVA. Differences in other parameters between different groups were analyzed using one-way ANOVA and Tukey's multiple comparison test. These analyses were performed using SPSS 24.0 software. A value of $\mathrm{P}<0.05$ was considered to be statistically significant.

\section{RESULTS}

\section{LRIC improved learning and memory ability in $2 \mathrm{VO}$ rats}

LRIC treatment was initiated on the third day after establishment of the $2 \mathrm{VO}$ model in rats. The Morris Water Maze was then used to test the effect of LRIC on cognitive impairment after $\mathrm{CCH}$ on the $21^{\text {st }}$ day, i.e., after 3 weeks of treatment. During the learning trials, the rats in all groups showed significant improvements in escape time latency ( $\mathrm{P}<0.01$, repeated-measures ANOVA). The mean escape latency over a period of 5 days for the $2 \mathrm{VO}$ group and the 2VO+LRIC group was significantly longer than the sham group, while the mean escape latency of 2VO+LRIC group was shorter than the $2 \mathrm{VO}$ group $(\mathrm{P}<0.05$, repeated-measures ANOVA; Fig. 2A). The Morris water maze test on day 6 showed that the percentage of time spent in the target quadrant by the $2 \mathrm{VO}$ group was significantly lower than that of rats in the sham group ( $\mathrm{P}<0.01$, Fig. 2B) and that LRIC treatment significantly increased the time in the target quadrant compared with the rats in the $2 \mathrm{VO}$ group $(\mathrm{P}<0.05)$.

\section{LRIC increased ATP and glucose in the cortex of $2 \mathrm{VO}$ rats}

The cerebral cortex is an area of vigorous energy metabolism and is responsible for cognitive function. Consequently, the effects of $\mathrm{CCH}$ are noted first in the associative cortical areas and spread diffusely thereafter via neuronal networks to impact the temporal and parietal cortices [33, 34]. With this pathogenic progression in mind, we selected the cerebral cortex as the region in which to record metabolic indices, such as glucose content and the ADP/ATP ratio. The ADP/ATP ratio in the cortex of $2 \mathrm{VO}$ rats was significantly higher than in the sham group while LRIC treatment for 1,2 and 4 weeks was found to significantly reduce the ratio of ADP/ATP in the cortex of $2 \mathrm{VO}$ rats $(\mathrm{P}<0.01, \mathrm{P}<0.001$ and $\mathrm{P}<0.05$, respectively, Fig. 3A-3C). Findings for glucose (GLU), which serves under normal physiological conditions as the main energy source for the brain, were similar [24]. As shown in Fig 2, GLU content in the cortex of 2VO rats was lower than that in the sham group while LRIC treatment for 1, 2 and 4 weeks significantly increased glucose content in $2 \mathrm{VO}$ rats $(\mathrm{P}<0.001, \mathrm{P}<0.001$ and $\mathrm{P}<0.05$, respectively, Fig. 3D-F). 

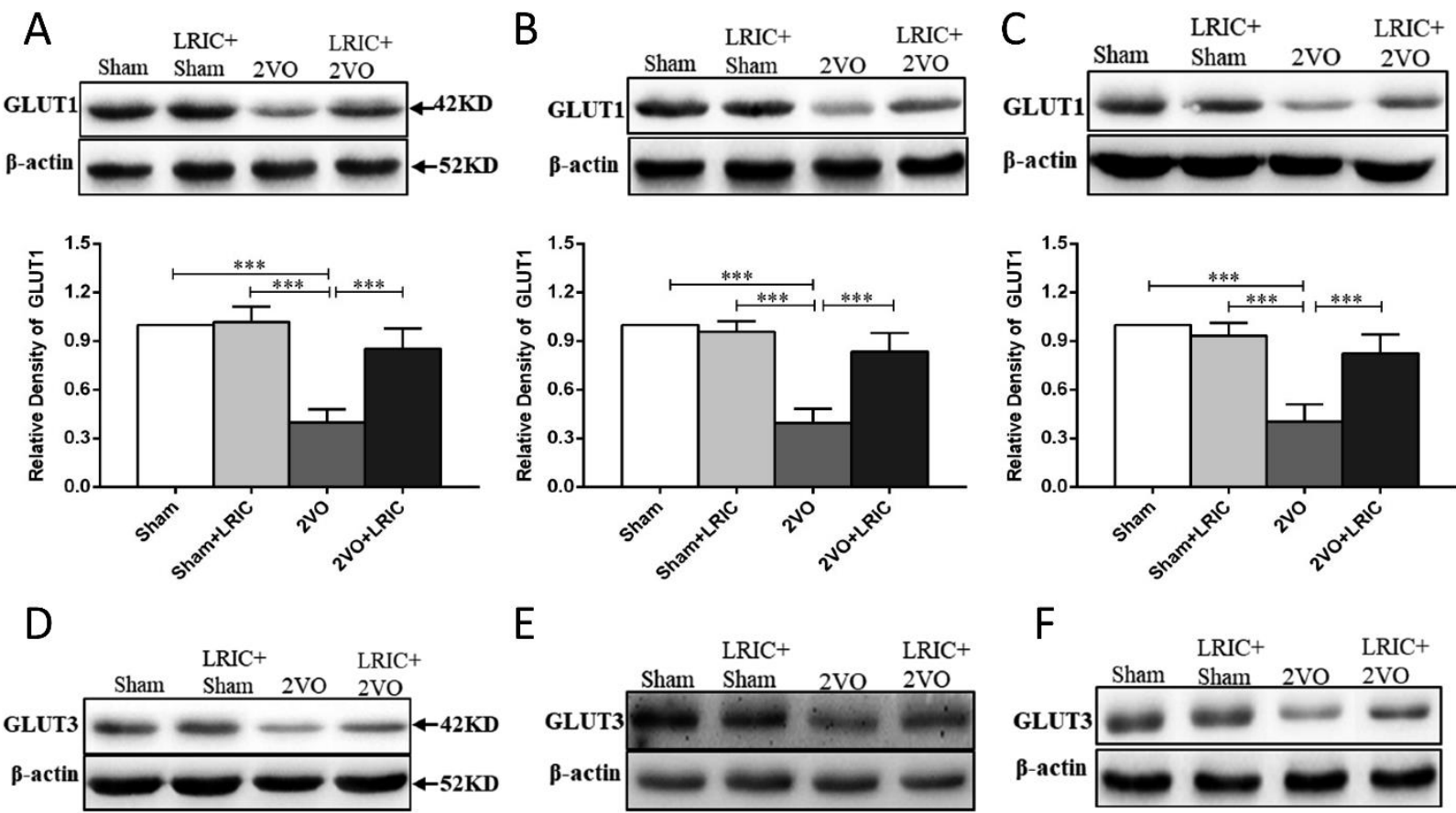

$\mathrm{E}$
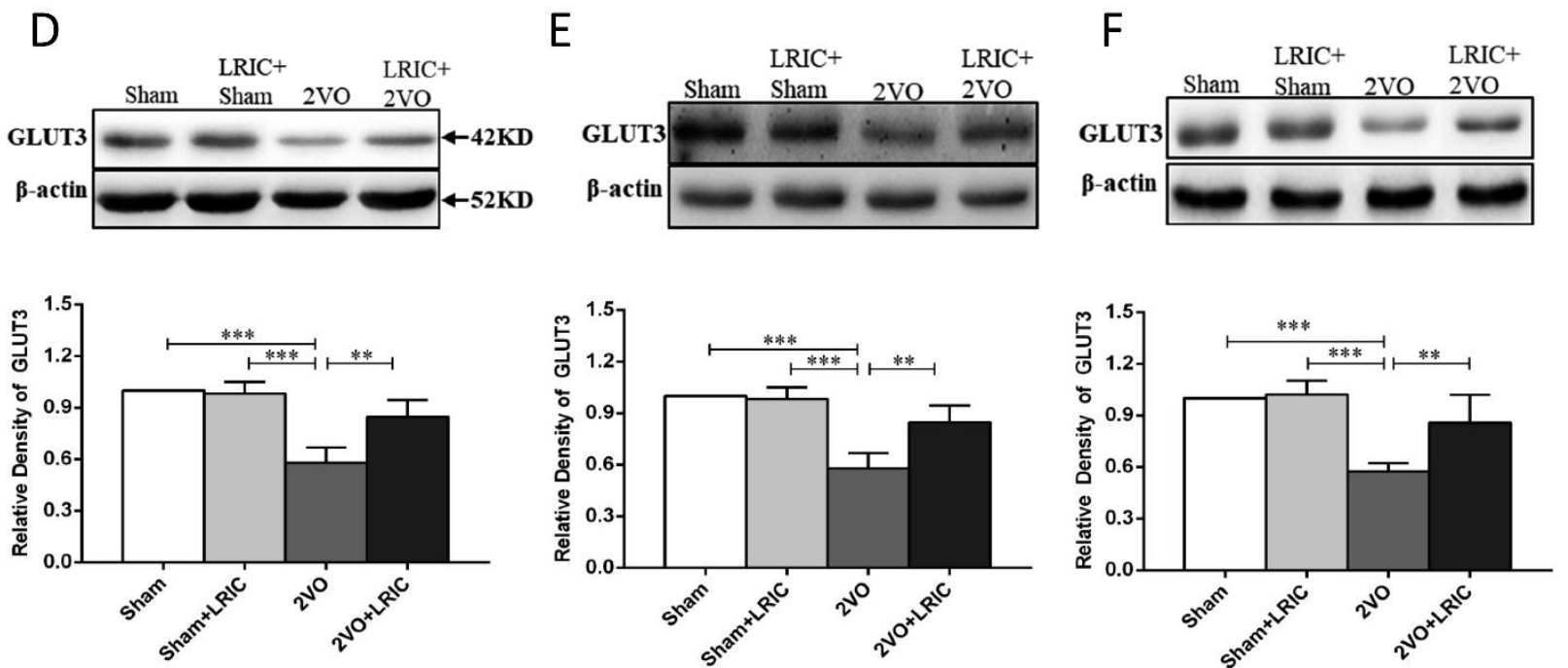

Figure 5. LRIC increased levels of GLUT1 and GLUT3 in the cerebral cortex as detected by Western blotting. (A and D) show levels of GLUT1 and GLUT3 at 1 week after treatment. (B and E) show levels of GLUT1 and GLUT3 at 2 weeks after treatment. (C and F) show levels of GLUT1 and GLUT3 at 4 weeks after treatment. $* P<0.05, * * P<0.01, * * * P<0.001$. Bar graphs are mean $\pm \mathrm{SD}, \mathrm{n}=10$ /group.

LRIC increased levels of glucose transport-related proteins PAMPKa, GLUT1 and GLUT3 in the cortex of $2 \mathrm{VO}$ rats

As assessed by Western blotting, levels of pAMPK $\alpha$ were significantly decreased in the cortex of $2 \mathrm{VO}$ rats and significantly increased after 1,2 and 4 weeks of LRIC treatment $(\mathrm{P}<0.001, \mathrm{P}<0.001$ and $\mathrm{P}<0.001$, respectively; Fig. 4A-C). We also measured the expression levels of GLUT1 and GLUT3 in the cortex using Western blot, finding that, when compared with the sham group, levels of GLUT1 and GLUT3 in the cortex of 2VO rats were significantly decreased. LRIC treatment for 1, 2 and 4 weeks increased levels of GLUT1 and GLUT3 compared with those found in the $2 \mathrm{VO}$ group $(\mathrm{P}<0.001$ for all, Fig. $5 \mathrm{D}-\mathrm{F})$. We further quantified the content of GLUT1 and GLUT3 in the cortex by ELISA at 4 weeks after LRIC treatment. Consistent with the Western blot results, LRIC significantly increased GLUT1 and GLUT3 levels as assessed by ELISA (Fig. 6A and B).

To investigate whether the increased levels of GLUT1 and GLUT3 obtained with LRIC treatment correlated with cognitive improvement, Pearson product linear regression analysis was performed. Results demonstrated that, after 4 weeks of LRIC treatment, the content of GLUT1 and GLUT3 in the cortex were negatively correlated with latency time (Figure 6C and D) and positively correlated with the percentage of time spent in the target quadrant during the Morris Water Maze test (Fig. 6E and F). These results suggest that upregulation of GLUT1/GLUT3 may be an important component of LRIC-induced cognitive improvement in the context of $\mathrm{CCH}$. 
A

C
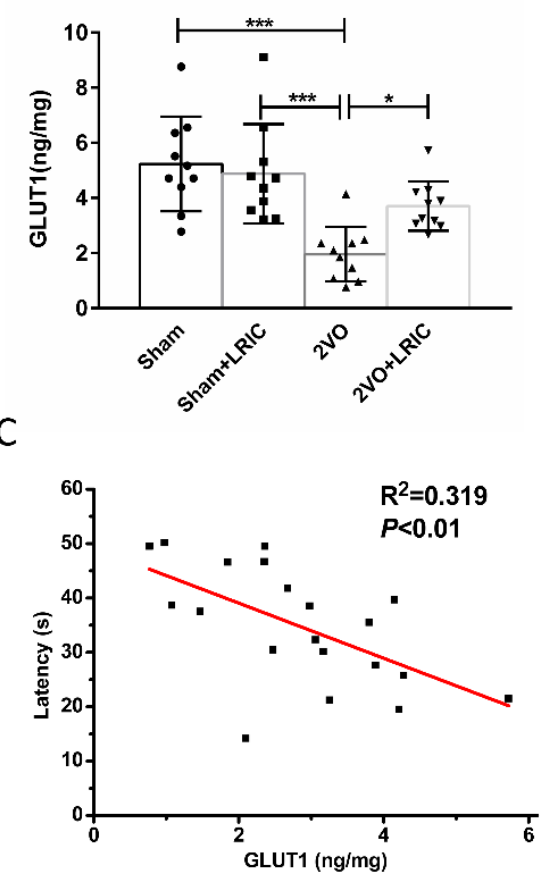

$E$

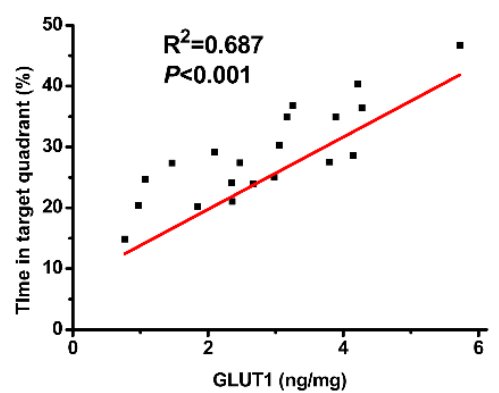

B

D
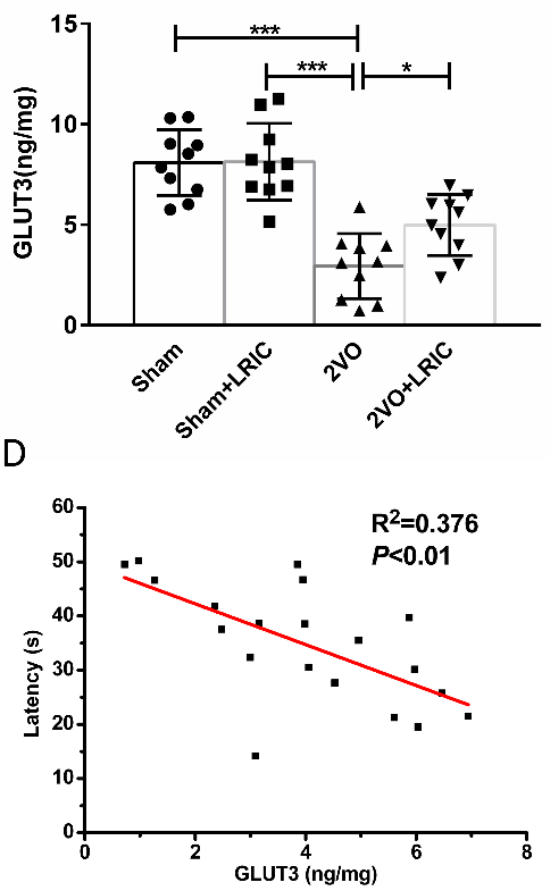

$\mathrm{F}$

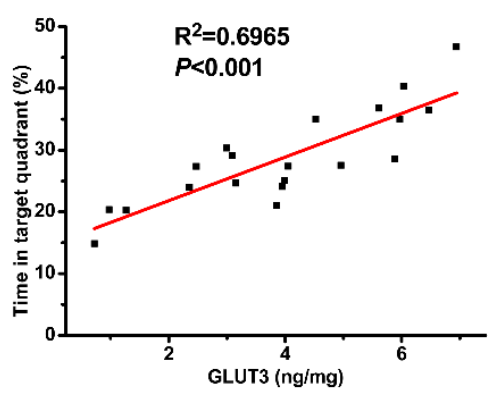

Figure 6. LRIC increases levels of GLUT1 and GLUT3 in the cerebral cortex as detected by ELISA. (A and B) show the data obtained by ELISA in rats at 4 weeks after treatment. $* P<0.05$, $* * * P<0.001$. Bar graphs are mean $\pm \mathrm{SD}, \mathrm{n}=10$ /group. (C and D) show the Pearson correlation between escape latency time on the fifth day of training and GLUT1 and GLUT3, respectively. ( $\mathbf{E}$ and $\mathbf{F}$ ) show the Pearson correlation between time in the target quadrant on the fifth day of training and GLUT1 and GLUT3, respectively.

\section{LRIC significantly increased expression of GLUT1 and GLUT3 in the cortical neurons of $2 \mathrm{VO}$ rats}

Neurons in the rat cortex were co-stained with GLUT1 or GLUT3 at 4 weeks after LRIC treatment initiation. GLUT1- or GLUT3-positive cells were stained red, NeuN-positive cells were stained green and GLUT1- or GLUT3- and NeuN-positive cells are shown in yellow (Fig. 7A and B). Compared with the sham group, expression levels of GLUT1 and GLUT3 in the cortical neurons of $2 \mathrm{VO}$ rats were decreased. In the LRIC group, expression levels of GLUT1 and GLUT3 in the cortical neurons of $2 \mathrm{VO}$ rats increased $(\mathrm{P}<0.05$ and $\mathrm{P}<0.05$, respectively; Fig. 7C and D).

Dorsomorphin abolished the beneficial effects of LRIC on learning and memory in $2 \mathrm{VO}$ rats
First, we found by Western blotting that inhibition of AMPK with dorsomorphin significantly decreased the expression of pAMPK $\alpha$ in both $2 \mathrm{VO}$ and LRIC-treated rats (Fig. 8A and 8B). LRIC without dorsomorphin improved the learning and memory ability of $2 \mathrm{VO}$ rats, but this effect was reversed with the administration of dorsomorphin (Fig. 8C and D); escape latency times were significantly increased in the LRIC+dorsomorphin group compared with the LRIC group $(\mathrm{P}<0.05)$. Correspondingly, the percentage of time spent in the target quadrant was significantly decreased in the LRIC+dorsomorphin group compared with the LRIC group $(\mathrm{P}<0.05)$.
Dorsomorphin abolished the effects of LRIC on glucose and ATP contents and on GLUT1 and GLUT3 expression levels


To explore whether dorsomorphin could inhibit the effects of LRIC on glucose and ATP contents, we measured glucose and ATP contents in the cortex after 4 weeks of LRIC treatment. Inhibition of AMPK with dorsomorphin significantly decreased both glucose and
ATP levels (Fig. 9A and B). We also quantified the expression levels of GLUT1 and GLUT3 in the cortex using ELISA after 4 weeks of LRIC treatment. Inhibition of AMPK with dorsomorphin significantly decreased the expression of GLUT1 and GLUT3 (Fig. 10A and 10B).
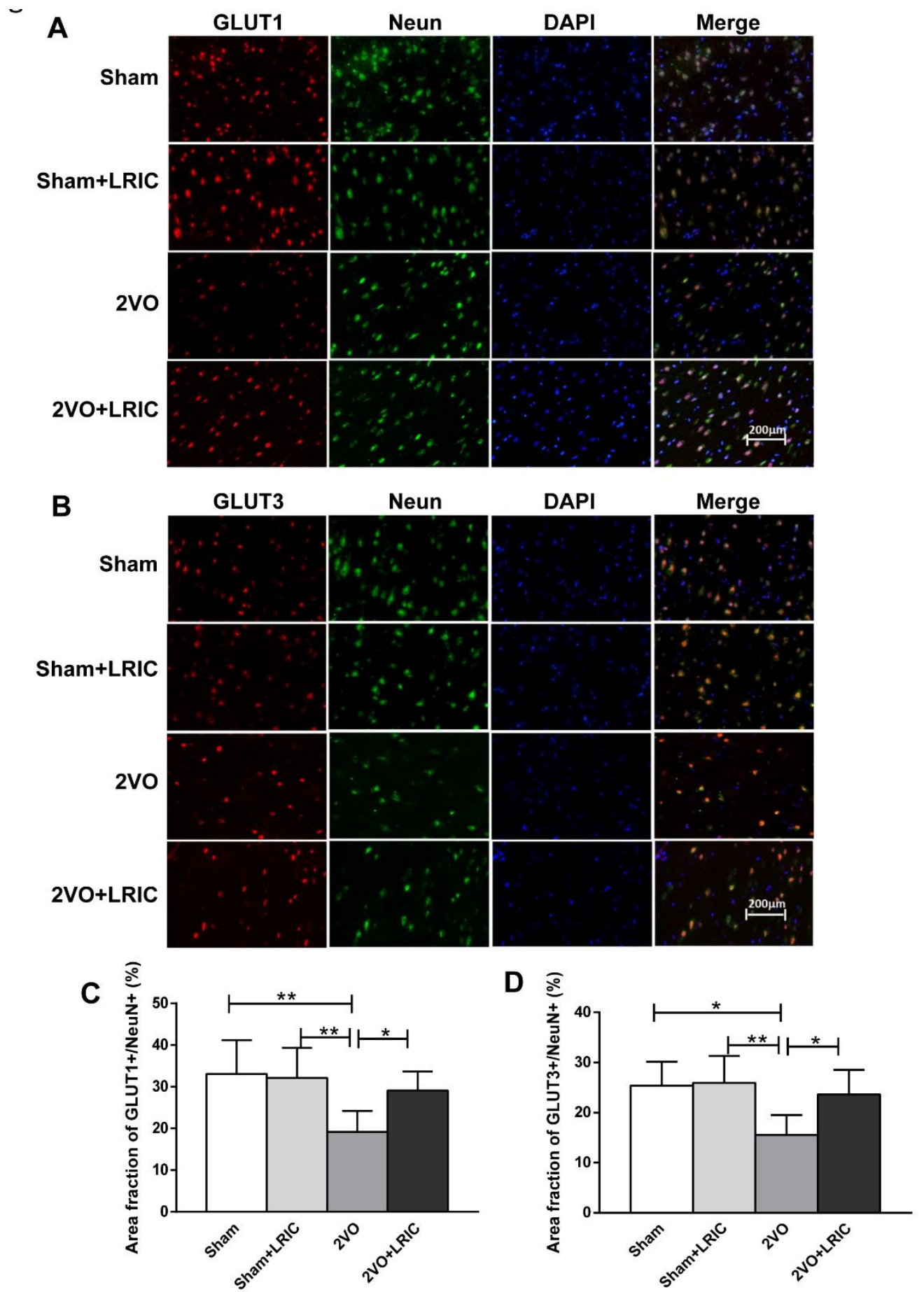

Figure 7. Immunofluorescence revealed that LRIC increased expression levels of GLUT1 and GLUT3 in the cortical neurons of $2 \mathrm{VO}$ rats. (A and B) show representations of immunofluorescence co-staining for GLUT1, GLUT3 and NeuN in the cerebral cortex. Scale bar=200 $\mu \mathrm{m}$. (C) Bar graphs show the areas of $\mathrm{GLUT}^{+} / \mathrm{NeuN}^{+}$. D. Bar graphs show the areas of $\mathrm{GLUT}^{+} / \mathrm{NeuN}^{+} . * * \mathrm{P}<0.01, * * * \mathrm{P}<0.001$. Data shown are mean $\pm \mathrm{SD}, \mathrm{n}=10 /$ group. 
A

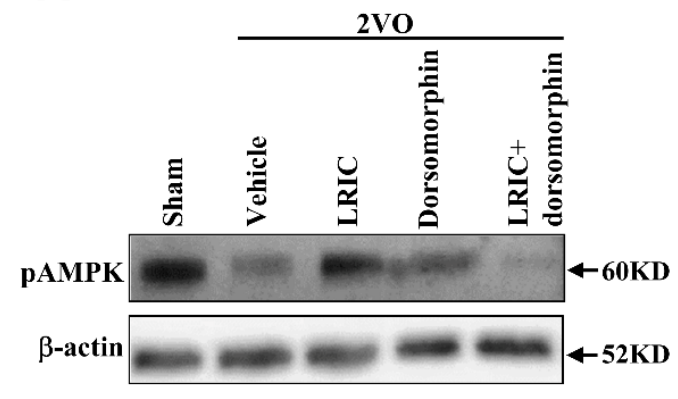

C

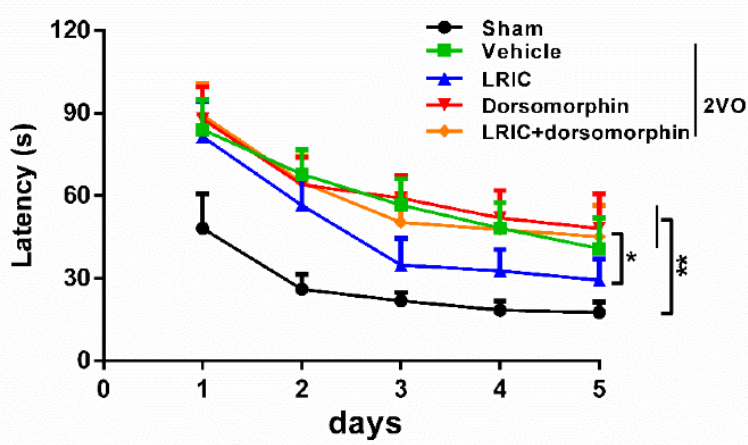

B

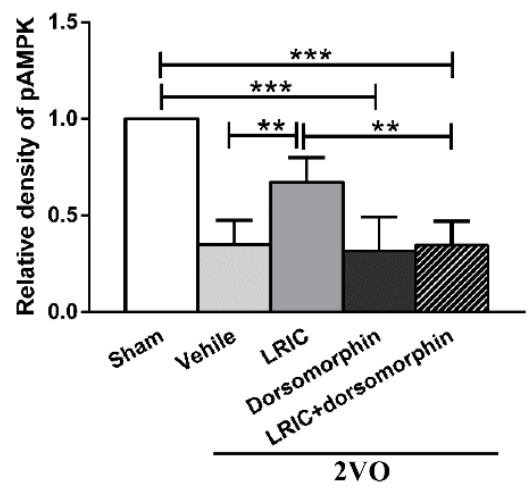

D

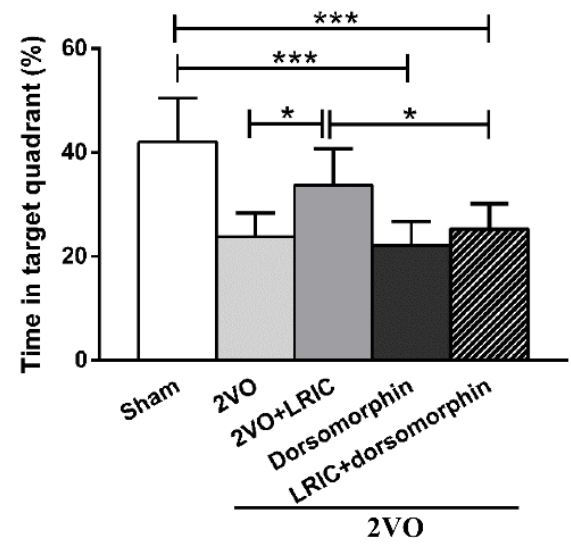

Figure 8. Dorsomorphin abolished the beneficial effects of LRIC on learning and memory in 2 VO rats. Intracerebroventricular administration of dorsomorphin decreased the expression of pAMPK $\alpha$ in the cortex at 4 weeks after treatment. (A) Representative Western blot bands. (B) Quantitative analyses of pAMPKa expression. $* * \mathrm{P}<0.01, * * * \mathrm{P}<0.001$. Data shown are mean $\pm \mathrm{SD}, \mathrm{n}=7 /$ group. (C) Escape latency time during the Morris water maze test after 3 weeks of LRIC treatment. (D) Percentage of time spent in the target quadrant. $* P<0.05$, $* * P<0.01$; $* * * P<0.01, \mathrm{n}=10 /$ group.

\section{DISCUSSION}

Here, we demonstrated that LRIC improved learning and memory after institution of a rat model of $\mathrm{CCH}$. We made the following novel observations: (1) LRIC effectively increased glucose and ATP levels in the CCH rat; (2) The improved glucose metabolism secondary to LRIC treatment was mediated in part through increased expression of pAMPK $\alpha$, GLUT1 and GLUT3; (3) Importantly, the expression levels of GLUT1 and GLUT3 in the cortex displayed a strong correlation with learning and memory as assessed using the Morris Water Maze test; (4) Treatment with dorsomorphin reversed the beneficial effects of LRIC on learning and memory, glucose and ATP contents and expression levels of GLUT1 and GLUT3. Taken together, these findings suggest that LRIC improved learning and memory in
$\mathrm{CCH}$ rats via improvements in AMPK-mediated glucose uptake.

Cognitive abilities, such as learning and memory, are closely linked with the level of glucose metabolism in the brain [35]. Different brain regions have different glucose consumption levels, with the cerebral cortex, medial and lateral geniculate bodies and thalamus exhibiting particularly robust rates [36]. Therefore, we chose the cerebral cortex as the location at which to evaluate the energy metabolism status of $2 \mathrm{VO}$ rats by measuring glucose content and found that the glucose content in the cortex of these rats decreased significantly. Since glucose is ultimately metabolized to produce ATP, which supplies energy for various brain activities [19], we also measured ATP content in rat cortical areas. As expected, the ATP content in the cortex of $2 \mathrm{VO}$ rats was also significantly reduced (i.e., exhibited an increased ADP/ATP ratio). 

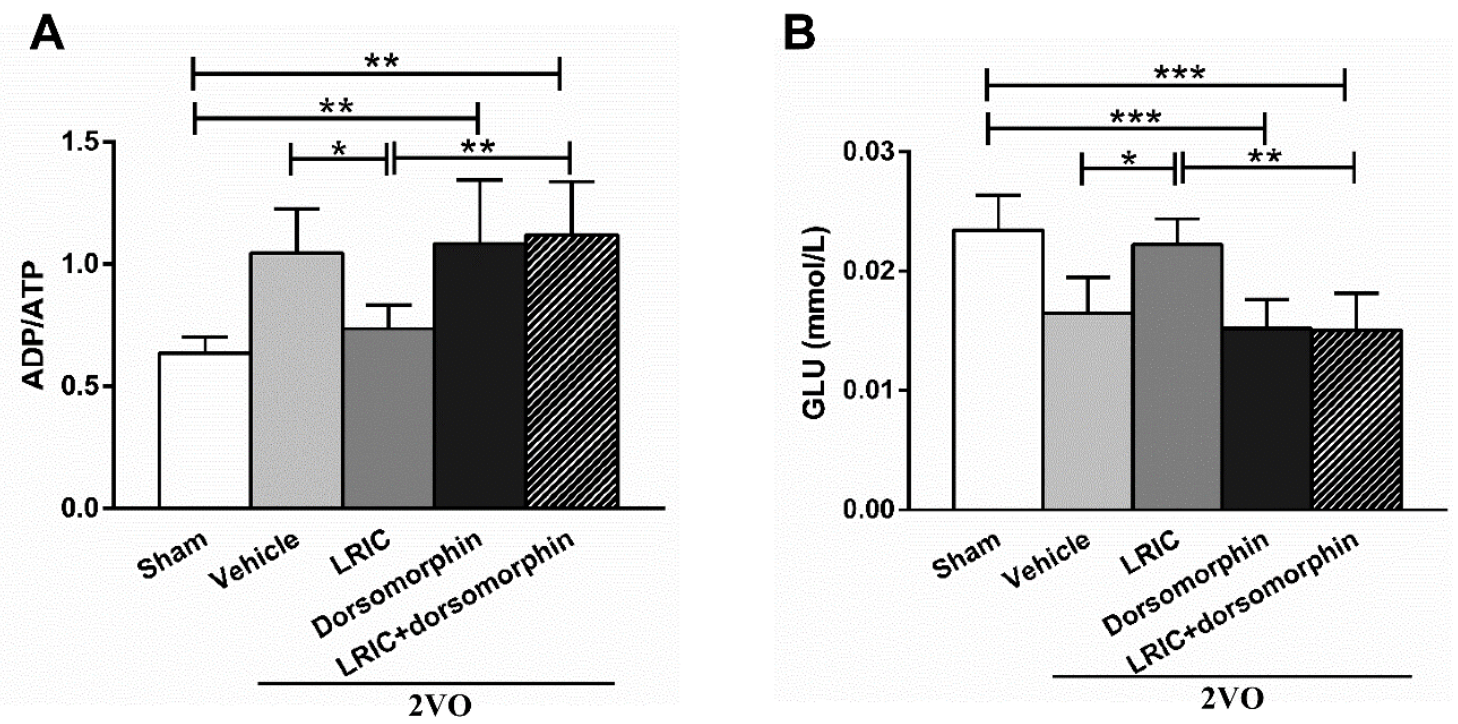

Figure 9. Dorsomorphin abolished the effect of LRIC on glucose and ATP contents. (A) This bar graph shows the ratio of ADP/ATP at 4 weeks after treatment initiation. (B) This bar graph shows glucose content at 4 weeks after treatment initiation. $* P<0.05, * * P<0.01, * * * P<0.001, \mathrm{n}=7 /$ group.

In recent years, LRIC has garnered increasing recognition as a novel treatment for cerebrovascular diseases, yielding protective effects against acute ischemic stroke [37], symptomatic intracranial atherosclerosis [6] and chronic cerebral ischemia [7, 14]. Despite these encouraging clinical results, however, the responsible mechanism remains unclear. A number of studies have attempted to investigate this mechanism, finding that LRIC can reduce the production of free radicals [38], promote cell survival pathways [39] and regulate the immune system [40]. Our study adds another contribution to this list by demonstrating that LRIC significantly increases glucose metabolism in the cortex of $2 \mathrm{VO}$ rats; compared with the $2 \mathrm{VO}$ group, the glucose and ATP content in the cortex of rats in the LRIC $+2 \mathrm{VO}$ group were significantly increased, while levels of lactic acid and pyruvate were significantly decreased. These results suggest that LRIC enhances cortical energy metabolism in $2 \mathrm{VO}$ rats by increasing the level of oxidative phosphorylation of glucose.
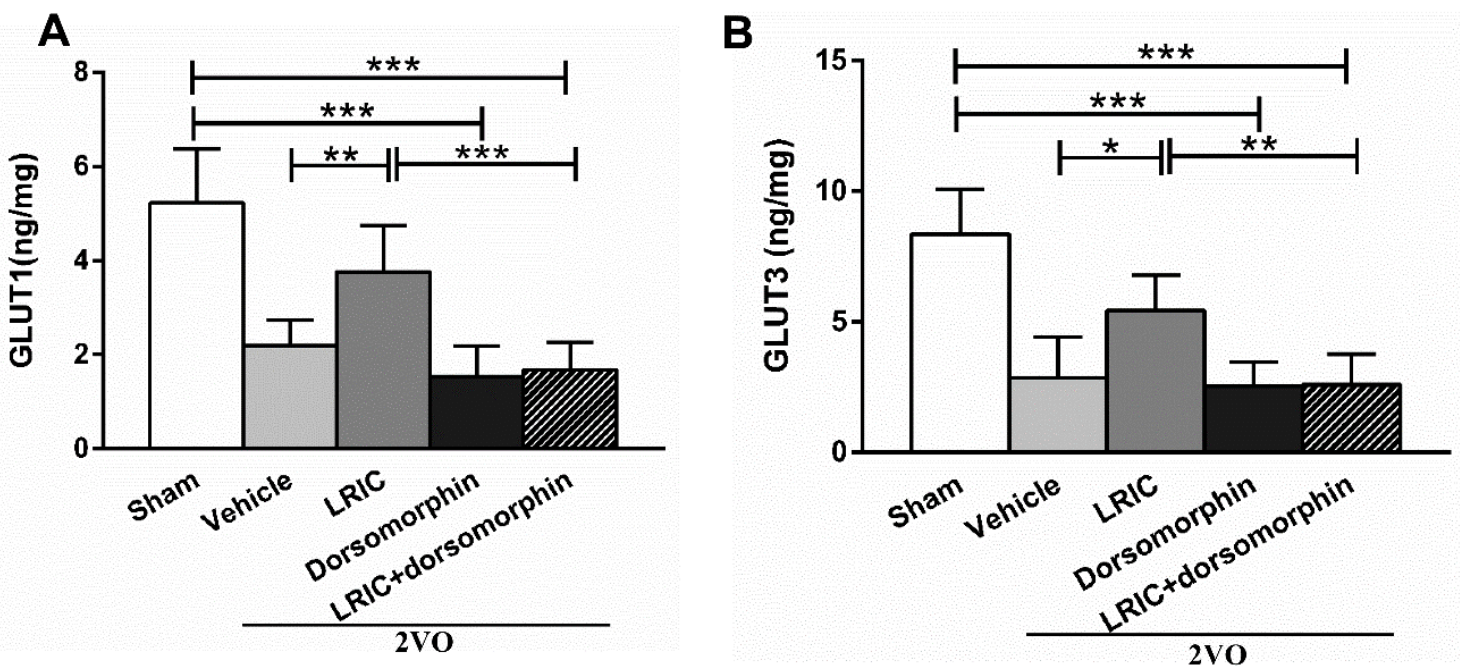

Figure 10. Dorsomorphin abolished the effect of LRIC on the expression of GLUT1 and GLUT3 detected by ELISA. (A) This bar graph shows the expression of GLUT1 after 4 weeks of treatment. (B) This bar graph shows the expression of GLUT3 after 4 weeks of treatment. $* P<0.05$, $* * P<0.01, * * * P<0.001, \mathrm{n}=7 /$ group. 
Maintenance of metabolic activity in mature brain tissue relies almost exclusively on the continuous oxidation of glucose [41]. Glucose is transported into the brain through the blood-brain barrier system by glucose transporters (GLUT) [42]. So far, 13 subtypes of GLUT have been found in mammals, of which GLUT1 and GLUT3 are widely expressed in the brain and considered the main glucose transporters in brain tissue [43]. Landau et al. reported that GLUT1 and GLUT3 expression was downregulated in the brain tissue of patients with Alzheimer's disease and glucose uptake was reduced, resulting in decreased cognitive function [44]. This link between glucose uptake and cognitive impairment has been linked to vascular insufficiency in recent studies showing that mice with reduced GLUT1 levels exhibit cognitive dysfunction associated with decreased brain capillary density, cerebral blood flow and glucose uptake, as well as increased blood-brain barrier leakage [21]. Similarly, studies utilizing knockout mice have confirmed that $\mathrm{GLUT1}^{+/-}$mice have reduced brain volume and abnormal motor behavior, while GLUT3 ${ }^{+/-}$mice exhibit abnormal spatial learning and working memory [45, 46]. In this study, immunofluorescence staining of the cortical neurons of $2 \mathrm{VO}$ rats for GLUT1 and GLUT3 revealed a significant decrease in the expression levels of these glucose transporters. Moreover, as the expression levels of GLUT1 and GLUT3 in the cortex displayed a strong correlation with learning and memory as assessed by the Morris water maze test, our findings suggest that GLUT1and GLUT3-regulated glucose metabolism in the cerebral cortex can play a key role in the cognitive function of $2 \mathrm{VO}$ rats.

Any factor that increases the ratio of AMP/ADP (such as starvation, oxidative stress, or glucose deprivation), can activate AMPK, which is responsible for the regulation of energy balance by acting on a number of downstream target proteins in multiple signaling pathways [47]. Specifically, studies have shown that AMPK serves as the molecular backbone of cellular energy metabolism, regulating intracellular energy processing by phosphorylation of metabolic enzymes and determination of GLUT gene expression throughout the brain [27]. It is also involved in the adaptation to cerebral ischemia and hypoxia; when these insults occur, increased expression of activated AMPK rapidly activates the glycolysis pathway in astrocytes to generate energy for neurons [48]. Our study found that pAMPK $\alpha$ levels were decreased in the cortical neurons of $2 \mathrm{VO}$ rats, but that LRIC significantly increased pAMPK $\alpha$ levels. Meanwhile, LRIC also significantly increased the expression levels of GLUT1 and GLUT3 in cortical neurons. These trends of change in pAMPK $\alpha$, GLUT1 and GLUT3 levels are consistent with those of glucose and ATP. Therefore, we hypothesized that pAMPK $\alpha$ may be an important regulator of cortical glucose metabolism in $2 \mathrm{VO}$ rats by augmenting GLUT1 and GLUT3 expression. To confirm this mechanistic link, a specific inhibitor of AMPK was administered; this intervention exerted a number of deleterious effects, including reversal of the neuroprotective effects of LRIC, as shown by increased escape latency time and reduced time in the target quadrant during the MWM test; increase in the cortical ratio of ADP/ATP and reduction of glucose content; and reduction of GLUT1 and GLUT3 expression levels.

In conclusion, the results of this study indicate that LRIC can increase the expression levels of GLUT1 and GLUT3 and increase glucose metabolism in the cerebral cortex by activating pAMPK $\alpha$, thereby improving the spatial learning and memory abilities of $\mathrm{CCH}$ rats. LRIC therefore appears to be a viable treatment to effectively improve cognitive function in $\mathrm{CCH}$. Given its safety, noninvasiveness and low cost, LRIC constitutes a promising potential treatment for patients with $\mathrm{VaD}$ and other cerebrovascular diseases entailing cognitive impairment. In the future, more research should be conducted to further evaluate the efficacy of this treatment to pave the way toward its application in $\mathrm{VaD}$ patients, for whom current treatments are very limited. On the other hand, these data also suggest that supplemental activation of glucose transport after $\mathrm{CCH}$ may provide a clinically applicable intervention for the promotion of cognitive impairment.

\section{Acknowledgments}

This work was supported by the Natural Science Foundation of Beijing, China (7161014). National Natural Science Foundation of China (81971114, 81801313, 81573867), the Beijing Municipal Administration of Hospitals' Youth Program (QML 20180801), the Science and Technology Development Project of the Beijing Municipal Health Commission and the National Key R\&D Program of China (2017YFC1308402).

\section{Conflict of interest}

The authors declare no conflict of interest

\section{References}

[1] Gorelick PB, Scuteri A, Black SE, Decarli C, Greenberg SM, Iadecola C, et al. (2011). Vascular contributions to cognitive impairment and dementia: a statement for healthcare professionals from the american heart association/american stroke association. Stroke, 42: 2672-2713

[2] Sato N, Morishita R (2013). Roles of vascular and metabolic components in cognitive dysfunction of 
Alzheimer disease: short- and long-term modification by non-genetic risk factors. Front Aging Neurosci, 5: 64

[3] Iadecola C, Duering M, Hachinski V, Joutel A, Pendlebury ST, Schneider JA, et al. (2019). Vascular Cognitive Impairment and Dementia: JACC Scientific Expert Panel. J Am Coll Cardiol, 73: 3326-3344

[4] Farooq MU, Min J, Goshgarian C, Gorelick PB (2017). Pharmacotherapy for Vascular Cognitive Impairment. CNS Drugs, 31: 759-776

[5] Hess DC, Blauenfeldt RA, Andersen G, Hougaard KD, Hoda MN, Ding Y, et al. (2015). Remote ischaemic conditioning-a new paradigm of self-protection in the brain. Nat Rev Neurol, 11: 698-710

[6] Meng R, Asmaro K, Meng L, Liu Y, Ma C, Xi C, et al. (2012). Upper limb ischemic preconditioning prevents recurrent stroke in intracranial arterial stenosis. Neurology, 79: 1853-1861

[7] Ren C, Li N, Li S, Han R, Huang Q, Hu J, et al. (2018). Limb Ischemic Conditioning Improved Cognitive Deficits via eNOS-Dependent Augmentation of Angiogenesis after Chronic Cerebral Hypoperfusion in Rats. Aging Dis, 9: 869-879

[8] Ren C, Li S, Wang B, Han R, Li N, Gao J, et al. (2018). Limb remote ischemic conditioning increases Notch signaling activity and promotes arteriogenesis in the ischemic rat brain. Behav Brain Res, 340: 87-93

[9] Wang Y, Meng R, Song H, Liu G, Hua Y, Cui D, et al. (2017). Remote Ischemic Conditioning May Improve Outcomes of Patients With Cerebral Small-Vessel Disease. Stroke, 48: 3064-3072

[10] Sun J, Tong L, Luan Q, Deng J, Li Y, Li Z, et al. (2012). Protective effect of delayed remote limb ischemic postconditioning: role of mitochondrial K(ATP) channels in a rat model of focal cerebral ischemic reperfusion injury. J Cereb Blood Flow Metab, 32: 851-859

[11] Wei D, Ren C, Chen X, Zhao H (2012). The chronic protective effects of limb remote preconditioning and the underlying mechanisms involved in inflammatory factors in rat stroke. PLoS One, 7: e30892

[12] Yang J, Liu C, Du X, Liu M, Ji X, Du H, et al. (2018). Hypoxia Inducible Factor 1alpha Plays a Key Role in Remote Ischemic Preconditioning Against Stroke by Modulating Inflammatory Responses in Rats. J Am Heart Assoc, 7

[13] Li X, Ren C, Li S, Han R, Gao J, Huang Q, et al. (2017). Limb Remote Ischemic Conditioning Promotes Myelination by Upregulating PTEN/Akt/mTOR Signaling Activities after Chronic Cerebral Hypoperfusion. Aging Dis, 8: 392-401

[14] Khan MB, Hafez S, Hoda MN, Baban B, Wagner J, Awad ME, et al. (2018). Chronic Remote Ischemic Conditioning Is Cerebroprotective and Induces Vascular Remodeling in a VCID Model. Transl Stroke Res, 9: 51-63

[15] Daulatzai MA (2017). Cerebral hypoperfusion and glucose hypometabolism: Key pathophysiological modulators promote neurodegeneration, cognitive impairment and Alzheimer's disease. J Neurosci Res,
[16] Hei Y, Chen R, Yi X, Long Q, Gao D, Liu W (2018). HMGB1 Neutralization Attenuates Hippocampal Neuronal Death and Cognitive Impairment in Rats with Chronic Cerebral Hypoperfusion via Suppressing Inflammatory Responses and Oxidative Stress. Neuroscience, 383: 150-159

[17] Wardlaw JM, Doubal F, Armitage P, Chappell F, Carpenter T, Munoz Maniega S, et al. (2009). Lacunar stroke is associated with diffuse blood-brain barrier dysfunction. Ann Neurol, 65: 194-202

[18] Yang Y, Rosenberg GA (2011). Blood-brain barrier breakdown in acute and chronic cerebrovascular disease. Stroke, 42: 3323-3328

[19] Benarroch EE (2014). Brain glucose transporters: implications for neurologic disease. Neurology, 82: 1374-1379

[20] Li W, Chaudhari K, Shetty R, Winters A, Gao X, Hu Z, et al. (2019). Metformin Alters Locomotor and Cognitive Function and Brain Metabolism in Normoglycemic Mice. Aging Dis, 10: 949-963

[21] Winkler EA, Nishida Y, Sagare AP, Rege SV, Bell RD, Perlmutter D, et al. (2015). GLUT1 reductions exacerbate Alzheimer's disease vasculo-neuronal dysfunction and degeneration. Nat Neurosci, 18: 521 530

[22] Bolanos JP, Delgado-Esteban M, Herrero-Mendez A, Fernandez-Fernandez S, Almeida A (2008). Regulation of glycolysis and pentose-phosphate pathway by nitric oxide: impact on neuronal survival. Biochim Biophys Acta, 1777: 789-793

[23] Wood IS, Trayhurn P (2003). Glucose transporters (GLUT and SGLT): expanded families of sugar transport proteins. Br J Nutr, 89: 3-9

[24] Camandola S, Mattson MP (2017). Brain metabolism in health, aging and neurodegeneration. Embo j, 36: 1474-1492

[25] Liu Y, Liu F, Iqbal K, Grundke-Iqbal I, Gong CX (2008). Decreased glucose transporters correlate to abnormal hyperphosphorylation of tau in Alzheimer disease. FEBS Lett, 582: 359-364

[26] Harr SD, Simonian NA, Hyman BT (1995). Functional alterations in Alzheimer's disease: decreased glucose transporter 3 immunoreactivity in the perforant pathway terminal zone. J Neuropathol Exp Neurol, 54: $38-41$

[27] Zhang Z, Liu H, Liu J (2019). Akt activation: A potential strategy to ameliorate insulin resistance. Diabetes Res Clin Pract, 156: 107092

[28] Vaibhav K, Braun M, Khan MB, Fatima S, Saad N, Shankar A, et al. (2018). Remote ischemic postconditioning promotes hematoma resolution via AMPK-dependent immune regulation. J Exp Med, 215: 2636-2654

[29] Farkas E, Luiten PG, Bari F (2007). Permanent, bilateral common carotid artery occlusion in the rat: a model for chronic cerebral hypoperfusion-related neurodegenerative diseases. Brain Res Rev, 54: 162180

[30] Jiang L, Cao Y, Liu Z, Ni S, Liu J, Ha Y, et al. (2020). 
SR $\mu$ CT Reveals 3D Microstructural Alterations of the Vascular and Neuronal Network in a Rat Model of Chronic Compressive Thoracic Spinal Cord Injury. Aging Dis, 11: 603-617

[31] Yu J, Wang WN, Matei N, Li X, Pang JW, Mo J, et al. (2020). Ezetimibe Attenuates Oxidative Stress and Neuroinflammation via the AMPK/Nrf2/TXNIP Pathway after MCAO in Rats. Oxid Med Cell Longev, 2020: 4717258

[32] Wang ZJ, Zhao F, Wang CF, Zhang XM, Xiao Y, Zhou F, et al. (2019). Xestospongin C, a Reversible IP3 Receptor Antagonist, Alleviates the Cognitive and Pathological Impairments in APP/PS1 Mice of Alzheimer's Disease. J Alzheimers Dis, 72: 1217-1231

[33] Volgyi K, Gulyassy P, Todorov MI, Puska G, Badics K, Hlatky D, et al. (2018). Chronic Cerebral Hypoperfusion Induced Synaptic Proteome Changes in the rat Cerebral Cortex. Mol Neurobiol, 55: 42534266

[34] Zadori D, Datki Z, Penke B (2007). [The role of chronic brain hypoperfusion in the pathogenesis of Alzheimer's disease--facts and hypotheses]. Ideggyogy $\mathrm{Sz}, 60$ : 428-437

[35] Ahmad W (2013). Overlapped metabolic and therapeutic links between Alzheimer and diabetes. Mol Neurobiol, 47: 399-424

[36] Roussel T, Frydman L, Le Bihan D, Ciobanu L (2019). Brain sugar consumption during neuronal activation detected by CEST functional MRI at ultra-high magnetic fields. Sci Rep, 9: 4423

[37] Che R, Zhao W, Ma Q, Jiang F, Wu L, Yu Z, et al. (2019). rt-PA with remote ischemic postconditioning for acute ischemic stroke. Ann Clin Transl Neurol, 6: 364-372

[38] Zhao H, Sapolsky RM, Steinberg GK (2006). Interrupting reperfusion as a stroke therapy: ischemic postconditioning reduces infarct size after focal ischemia in rats. J Cereb Blood Flow Metab, 26: 11141121

[39] Gao X, Zhang H, Takahashi T, Hsieh J, Liao J, Steinberg GK, et al. (2008). The Akt signaling pathway contributes to postconditioning's protection against stroke; the protection is associated with the MAPK and PKC pathways. J Neurochem, 105: 943955

[40] Xing B, Chen H, Zhang M, Zhao D, Jiang R, Liu X, et al. (2008). Ischemic post-conditioning protects brain and reduces inflammation in a rat model of focal cerebral ischemia/reperfusion. J Neurochem, 105: 1737-1745

[41] Jolivet R, Magistretti PJ, Weber B (2009). Deciphering neuron-glia compartmentalization in cortical energy metabolism. Front Neuroenergetics, 1: 4

[42] Correia SC, Santos RX, Perry G, Zhu X, Moreira PI, Smith MA (2011). Insulin-resistant brain state: the culprit in sporadic Alzheimer's disease? Ageing Res Rev, 10: 264-273

[43] Leino RL, Gerhart DZ, van Bueren AM, McCall AL, Drewes LR (1997). Ultrastructural localization of GLUT 1 and GLUT 3 glucose transporters in rat brain. J Neurosci Res, 49: 617-626

[44] Jagust WJ, Bandy D, Chen K, Foster NL, Landau SM, Mathis CA, et al. (2010). The Alzheimer's Disease Neuroimaging Initiative positron emission tomography core. Alzheimers Dement, 6: 221-229

[45] Tang M, Gao G, Rueda CB, Yu H, Thibodeaux DN, Awano T, et al. (2017). Brain microvasculature defects and Glut1 deficiency syndrome averted by early repletion of the glucose transporter-1 protein. Nat Commun, 8: 14152

[46] Zhao Y, Fung C, Shin D, Shin BC, Thamotharan S, Sankar R, et al. (2010). Neuronal glucose transporter isoform 3 deficient mice demonstrate features of autism spectrum disorders. Mol Psychiatry, 15: 286299

[47] Day EA, Ford RJ, Steinberg GR (2017). AMPK as a Therapeutic Target for Treating Metabolic Diseases. Trends Endocrinol Metab, 28: 545-560

[48] Curry DW, Stutz B, Andrews ZB, Elsworth JD (2018). Targeting AMPK Signaling as a Neuroprotective Strategy in Parkinson's Disease. J Parkinsons Dis, 8: 161-181 